\title{
A new era for executive function research: On the transition from centralized to distributed executive functioning
}

\author{
Nicolas Zink ${ }^{1 \#}$, Agatha Lenartowicz ${ }^{1}$, Sebastian Markett ${ }^{2}$ \\ ${ }^{1}$ Department of Psychiatry and Biobehavioral Sciences, University of California, Los Angeles, \\ Los Angeles \\ ${ }^{1}$ Department of Psychology, Humboldt University Berlin, Berlin, Germany \\ \# Corresponding author \\ Nicolas Zink
}




\begin{abstract}
"Executive functions" (EFs) is an umbrella term for higher cognitive functions such as working memory, inhibition, and cognitive flexibility. These functions refer to dissociable mechanisms that are also intricately related, justifying the view of EF as a unitary mental faculty. One of the most challenging theoretical problems in this field of research has been to explain how the wide range of cognitive processes subsumed as EFs are controlled without an all-powerful but ill-defined central executive in the brain. Efforts to localize control mechanisms in circumscribed brain regions have not led to breakthrough in understanding how the brain controls and regulates itself, and no single brain system underlying a 'central executive' has yet been identified. We discuss how a distributed control network view can help to refine our understanding of the neurophysiological mechanisms underlying EFs. In this view, executive control functions are realized by spatially distributed brain networks, thus precluding the need for a modular central executive. We further discuss how graph-theory driven analysis of brain networks offers a unique lens on this problem by providing a reference frame to study brain connectivity in EFs in a holistic way and how neuroscience network research endeavors to investigate clinical neuropathology of disrupted EFs.
\end{abstract}

\title{
Introduction
}

The ability to exert control over actions is essential for personal autonomy and one of the most impressive yet poorly understood capacities our brains endow us with. The processes that control actions cover a range of cognitive skills referred to as executive functions $(\mathrm{EFs})^{1,2}$. While the precise definition of EFs remains a topic of debate ${ }^{3}$, there is a common consent in terms of the importance of EFs for adaptive behavior in an ever-changing environment ${ }^{4-6}$. Numerous theoretical frameworks exist to classify different EFs and to describe how they work together ${ }^{1,3,5,7-9}$. According to Miyake ${ }^{9}$ and Diamond $^{1}$, at least three core functions of EF can be identified, which are likely to represent distinct cognitive subsystems that nevertheless share a functional overlap to some degree: updating and monitoring of working memory representations, inhibition and interference control, as well as cognitive flexibility and shifting. Working memory (WM) is a cognitive system that enables to manipulate and maintain restricted chunks of information which are stored in short-term memory $1,10-$ ${ }^{14}$. Inhibition has been defined as "being able to control one's attention, behavior, thoughts, and/or emotions to override a strong internal predisposition or external lure, and instead do what's more appropriate or needed"1 ${ }^{1}$. Cognitive flexibility reflects the ability to consider multiple conflicting representations of a single object or event simultaneously ${ }^{15}$ and selectively switch between actions, perspectives, and strategies for appropriate action in a changing environment ${ }^{1,16}$. In addition to flexibly switching between several laboratory tasks, every-day life as well as modern work environments often require performance on two or more tasks at the same time, e.g. driving while having a telephone conversation or simultaneously controlling several displays in air traffic control. In this multitasking context, efficient EFs are essential to select relevant information from the environment and to adjust performance to environmental demands by flexibly shifting between more serial or more parallel processing strategies ${ }^{17}$.

\section{Centralized Control in Cognition and Cognitive Neuroscience}

Substantial theoretical and experimental progress has been made in the past 50 years to describe how humans control their actions by selecting, processing, and prioritizing task-relevant stimuli (often at the expense of simultaneously present yet less relevant signals), and flexibly adapt to changing demands from the environment ${ }^{5,18-24}$. Early cognitive models have introduced a hypothetical central executive to their cognitive architectures ${ }^{5,12}$. One unifying premise underlying these theories is that some sort of central executive system is required that governs exerted control by "dampening" irrelevant and prioritizing relevant salient information or schemes. But while the necessity of higherorder control functions that control lower-order functions is self-evident, it does not explain how this central executive operates, reducing instead to the question of who is in charge of controlling the central executive. This problem of self-recurrency has been widely acknowledged and has led to the criticism that the central executive resembles an all-powerful but ill-defined "homunculus" that directs all processes that are not "automatic" 25 . Any theoretical framework of EFs must therefore not only 
specify how control operates, how it is implemented at the neurophysiological level, and how it integrates other cognitive processes and their interrelatedness, but also formally address the question of what is controlling the controller in order to avoid an infinite hierarchy of central executives or homunculi ${ }^{27}$.

One of the most influential theoretical frameworks on the neurophysiological mechanisms of EFs that has pushed beyond the central executive view has been proposed by Miller and Cohen in $2001^{28}$. This framework emphasizes the importance of the prefrontal cortex (PFC) as a key region to implement a wide range of EFs through active maintenance of goals and the means to achieve them. Rooted in the biased-competition model of selective attention ${ }^{29}$, it cast the problem of control as a case of prioritization of competing perceptual or response representations. The role of the PFC is to bias activity in regions responsible for those representation, in accordance with a behavioral goal, that it maintains in memory. Thus, the PFC indirectly acts as a central executive and intervenes in a top-down fashion by selectively prioritizing the relevant process. According to this perspective, exerted control in $\mathrm{EF}$ is domain-general and operates on specific subordinated cognitive functions in service of a higherorder task goal. EFs such as inhibition, working memory and cognitive flexibility emerge from the dynamics of the PFC biasing goal-relevant activity patterns in the brain. In the last 20 years, this theoretical model has provided a versatile framework that has generated much empirical evidence supporting the pivotal role of the PFC in many EFs. However, despite progress in understanding the neurophysiological mechanisms of how the PFC represents and implements behavioral goals, the mechanism by which it accounts for EFs remains elusive. Although the PFC may participate to a greater extent than other areas in EFs, increasing evidence suggests that it does not act alone. Damage to the PFC, for instance, may or may not be accompanied by deficits in EFs, and neither site nor size of a prefrontal lesion allow for a clear prediction about the nature of resulting problem in executive functioning ${ }^{30}$.

In parallel, the advance of non-invasive neuroimaging techniques such as $\mathrm{fMRI}$, in combination with experimental designs rooted in subtractive logic ${ }^{31,32}$, contributed to the conceptualization of a modular brain architecture ${ }^{33-36}$. The results could be construed as a fractionalization of the central executive ${ }^{1,28,37-52}$, now a "macroconstruct" in which the central executive consists of discrete subsystems with anatomically segregated and functionally specialized modules. In a typical neuroimaging study, the cognitive process of interest is isolated via correlation to an external manipulation thought to elicit that process, and associated with a brain region of interest. If this association is statistically significant, it is assumed that this process is located within this brain area. This strategy has ascribed different aspects of EFs with specific anatomically circumscribed brain areas. For instance, goal-directed behavior with the ventromedial prefrontal cortex $\left(\mathrm{vmPFC}^{53-59}\right)$ and dorsolateral prefrontal cortex (dIPFC ${ }^{55-57,60,61}$ ), inhibition with right ventrolateral prefrontal cortex (vIPFC ${ }^{62-65}$ ), conflict monitoring, selective attention, and computation of the expected value of control with anterior cingulate cortex $\left(\mathrm{ACC}^{39,49,50,58,60,66-77}\right)$, and cognitive flexibility and voluntary motor action selection with basal ganglia $\left(\mathrm{BG}^{78-83}\right)$. The problems with such an approach have been extensively discussed and include subsequent tendencies towards reverse inference and inherent bias in interpretation ${ }^{84,85}$. Furthermore, a modular perspective fails to appreciate the activity of this region in the context of the other activities within the brain. The systematic association of a region with an EF such as inhibition could therefore reflect an emergent property of a broader neural network (e.g., involved in detection of salient cues, such as for inhibition) $)^{65}$, and thus risks to falsely localize higherlevel cognitive processes at a lower level of processing ${ }^{86}$, especially in highly connected regions such as the ACC and basal ganglia.

Although it is intuitive to assume that conceptually distinct EFs must also have distinct anatomical representations ${ }^{87}$, attempts to identify neuroanatomical correlates of the central executive ${ }^{1,28,37-52}$ have not yet led to breakthroughs to describe their neurophysiological mechanisms. The idea of subsystems that provide specialized, subsidiary central executive functions is challenged by empirical evidence suggesting that the same putative brain correlates for EFs such as the PFC and ACC have been 
associated with functionally different $\mathrm{EFs}^{39,40,49,50,53,54,66,68,70,72-76,88-93}$. This raises the question how a plethora of diverse EFs can arise from a rather static neuroanatomical architecture ${ }^{94}$. In complement, different EFs such as inhibition, flexibility, and working memory have been shown to activate large proportions of the cerebrum and converge within PFC, ACC, and posterior parietal cortex ${ }^{95}$. Moreover, the putative brain areas underlying EFs also show large functional overlap, such as in goal directed behavior $\left(\mathrm{ACC}^{70}\right.$; dIPFC ${ }^{55-57,61}$; vmPFC $\left.{ }^{53,55-57,61}\right)$, prediction and evaluation of an action $\left(\mathrm{ACC}^{49,50,58,66-68}\right.$; vmPFC $\left.{ }^{58}\right)$, and selective attention $\left(\mathrm{ACC}^{60,74,75,77}\right.$; $\mathrm{vmPFC}^{59}$; dIPFC ${ }^{60}$ ), which challenges the functional specificity of these regions. Arguably, the focus on activity of specific regions of interests or single event-related components has therefore substantially limited the possibility to identifying the neural correlates of EFs from a whole brain perspective.

Increasing evidence suggests that these putative brain areas underlying EFs seem to interact. The ACC, for instance, has been found to support PFC in maintaining and updating task-relevant representation in working memory ${ }^{69,96,97}$ and signal the need for control ${ }^{39,52}$. Furthermore, studies indicate that cognitive flexibility is orchestrated within $\mathrm{BG}^{83}$, but also supported by $\mathrm{PFC}^{78,79}$ and $\mathrm{ACC}^{98}$. Similarly, close interrelation between PFC and BG were found to support WM processes ${ }^{99-106}$. Thus, once again, the data suggest that pivotal brain areas that contribute significantly to EFs, such as PFC, ACC, and basal ganglia, act within spatially distributed networks and include other structures that may also be relevant to explain EFs. This point is echoed in studies of neuropsychiatric disorders that exhibit impairments in EFs, and that reveal aberrant development and functional connectivity in spatially distributed brain networks ${ }^{107-146}$.

Thus, mounting evidence suggest that there is no single brain region that sits at the control apex, and, furthermore, efforts to define EF-dedicated neuroanatomical substrates have not been conclusive. The question of how EFs are generated by the brain persists. In the following sections we consider an alternate view to centralized or localized EFs. We argue based on suggestions from the cognitive and computational literature ${ }^{86,147,148}$, and in line with the recognized significance of functional connectivity patterns to neural organization and function ${ }^{149-152}$, that EFs are neither a strictly top-down generated process nor one that can be localized in specific brain correlates, but rather that EFs are the emerging consequence of communication within a broad network of spatially and functionally dispersed brain areas that integrate different aspects of EFs.

\section{Distributed Control and Executive Functions}

A core assumption underlying many theoretical frameworks on EFs is of hierarchical architecture, namely, that brain areas or circuits underlying EFs regulate, but do not participate in the more basic cognitive processes that they control ${ }^{28,38}$ (Figure 1A). This top-down view, by design, relies on a centrally operating executive. However, Barnard and Bowman (2004), Vandierendonck (2016) and Eisenreich et al. (2017), have argued that EFs can also be a result of a conceptually and anatomically distributed control system (DCS ${ }^{86,147,148}$. One such DCS view is inspired from early connectionist models ${ }^{153,154}$, in which control and controlled processes are co-localized within large numbers of dispersed elements (Figure 1B). In contrast to a hierarchical architecture where core brain regions are assumed to solely exert control on more basic processes, in a DCS, each element can be a controller and an element that is being controlled. In computational models of distributed control, the state of the overall system is often independent from any constituting element ${ }^{147}$. In other words, in such systems, behavioral control is analogous to the state of the overall system, and is therefore an emergent function arising from the interaction of all elements ${ }^{155,156}$. Recent evidence of distributed control in nonbrain biological systems such as in schools of fish, flocks of birds, swarms of insects ${ }^{86,157,158}$, and herds of baboons s $^{159,160}$ and in distributed deep learning algorithms ${ }^{161}$ highlight the strength and plausibility of a DCS view. Apparent control in these biological distributed systems is often realized via a couple of simple rules that guide the indirect coordination between agents or actions. In schools of fish or flocks of birds, the control over the shape of the swarm and the moving direction is a consequence of the distance kept between adjacent agents and the rule about when to change 
direction (i.e. follow the group average ${ }^{160}$ ). These rules enable fast communication of information (e.g. about the direction of movement) across all agents, without reliance on a top-down controller.

A
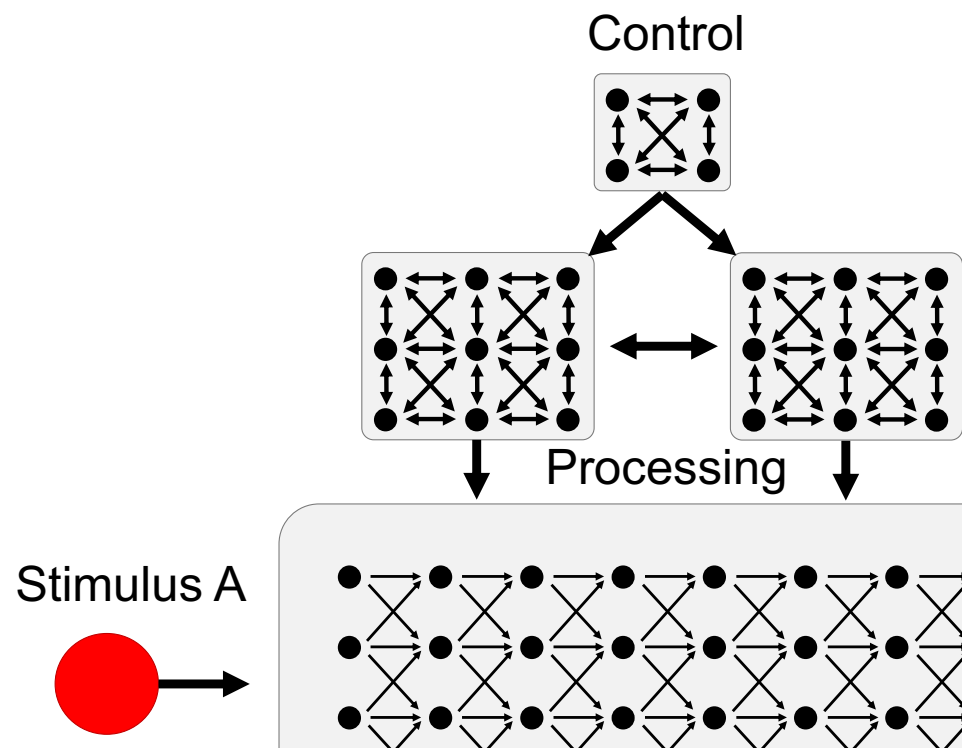

Stimulus B
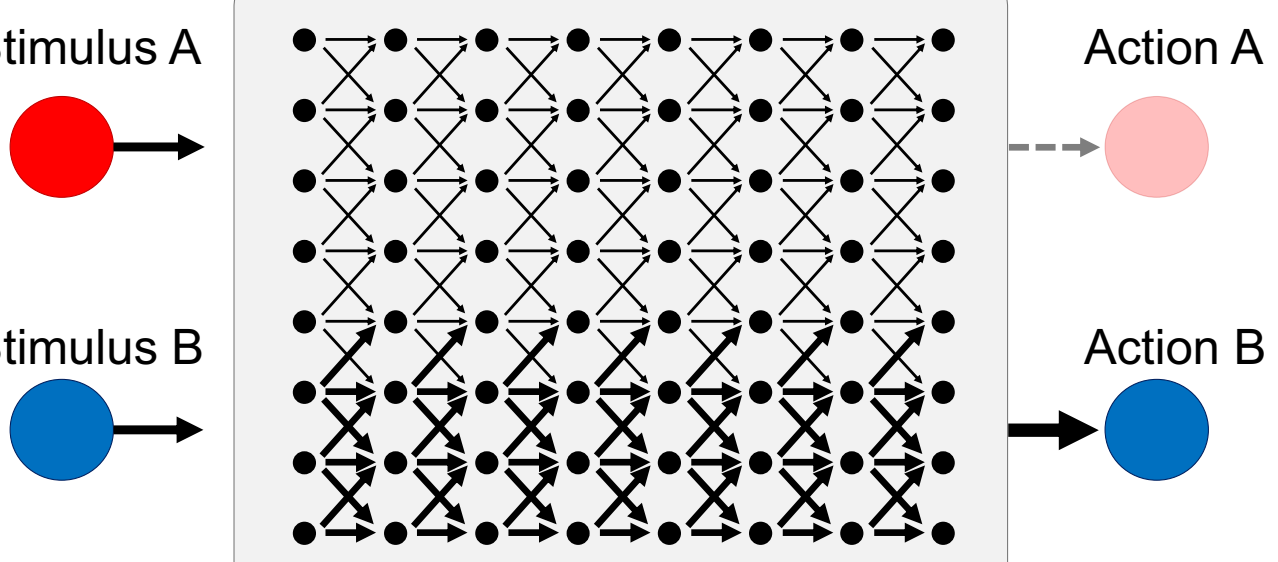

B

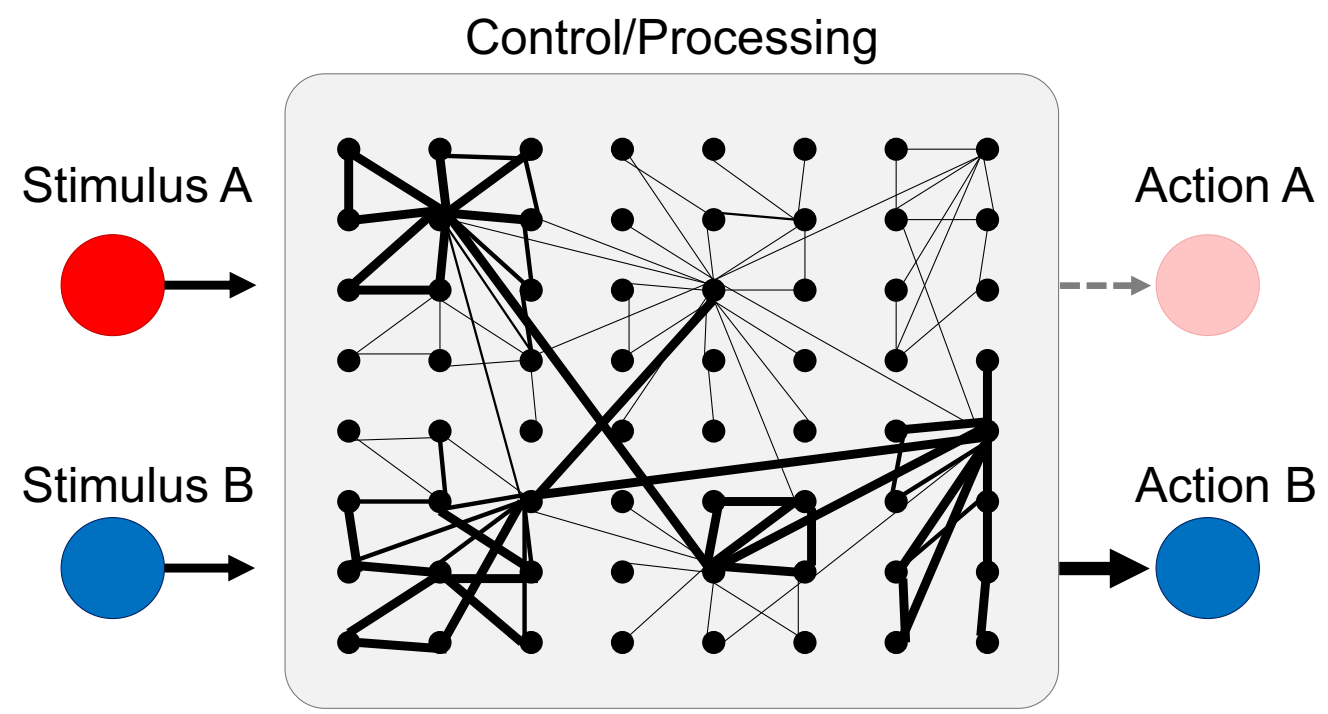

Figure 1. Organization of a modular executive control network vs. a distributed control network, in which two stimuli (stimulus A and stimulus B) lead to an action outcome (action A vs. action B). A. Top- 
down controlled, hierarchically organized control-modules govern automatized processing. In this system, control and processing elements are distinct and localized to specific areas. B. Distributed control networks, in which control and processing elements are combined in individual agents. These agents form individual clusters. Adapted and modified from Eisenreich et al. (2017) 230 .

Can a DCS framework explain EFs? EFs can be conceptualized as a consequence of the interaction between distributed elements, thus avoiding the problem of any central executive or central executive subsystem. Increasingly evidence suggests that, indeed, properties of EF generation in the brain are consistent with properties of a DCS. One such principle is that all elements can be controlled or be a controller, or, in other words, the emergent property can be observed in any subset of the system. Several studies have highlighted that prefrontal regions, a putative apex of the central executive, as well as posterior regions seem to exhibit both basic and control processing ${ }^{162-165}$. In line with the DCS view, it has been suggested that working memory (WM), a core EF, is a general property of the cortex, not limited to specific regions ${ }^{164,166}$. This proposal shifts perspective of WM from localized to a highly distributed process wherein information can be maintained in any system engaged in the initial perceptual processing, including any region contributing to the initial percept ${ }^{166}$. The second key principle of a DCS is the existence of local rules capable of generating the emergent phenomenon, such as the distance kept between adjacent agents in a swarm. In the brain, from single neuron firing to neuron populations and between brain area communications, each level is a complex function of its lower level constituents and embedded in a larger-scale organization ${ }^{167}$. In this context, EFs such as the initiation vs. the inhibition of an action or switching to another action might be the emergent behavioral outcome from simpler local neurophysiological mechanisms of the participating brain areas, for instance via neuronal interactions resulting in local gain modulation ${ }^{168-172}$ and the collective state of each contributing neuron ${ }^{167}$. In particular, evidence supporting biased competition among neuronal populations in generating initiation or inhibition in selective attention ${ }^{29,173}$, suggests that such competition is a general property of neurons across the brain, not limited to any central controller. From this perspective, the emergence of executive control patterns (e.g. initiating or inhibiting an action or switching to another action) observed in putative control structures like the PFC, ACC, and basal ganglia may reflect the overall state of the system, in the same way a single fish in a swarm or a single bird in a flock reflects the state of the overall system (i.e. the trajectory of the movement), without the requirement to be on top of the hierarchy in the control system.

Another key property of a DCS is its robustness to perturbations. In contrast to centralized systems, in which a nonbrain biological systems such as swarm would be vulnerable to the loss of its leading agent, a swarm organized as a DCS has been shown to be robust to degradation ${ }^{174}$. Similarly, decentralized (i.e. distributed) networks have been shown to be resilient systems which are capable of absorbing large external perturbations without undergoing functional breakdown ${ }^{167,175-177}$. A DCS network organization in the brain may therefore explain how EFs can be preserved to some extent in the face of pathological attack by lesion and substance-related disorders ${ }^{178-180}$, neuropsychological disorders like ADHD $^{181-184}$, schizophrenia ${ }^{185-188}$, and restless legs syndrome ${ }^{189}$, aberrant development such as in autism ${ }^{120,190}$, and cognitive decline such as Alzheimer's ${ }^{191-195}$ and Parkinson's disease ${ }^{196,197}$. As noted previously, damage to the PFC, as well, may or may not be accompanied by deficits in EFs, and neither site nor size of a prefrontal lesion allow for a clear prediction about the nature of resulting problem in executive functioning ${ }^{30}$. Thus, EFs do exemplify some robustness in the human brain. One way to investigate the robustness of EFs as a DCS is using 'lesioned' networks in computational models, which investigate how an observed profile of anatomical or functional dysconnectivity in a mature network might have been generated by earlier developmental abnormalities ${ }^{175,198-201}$. In order to explore the effect of acute damage on overall performance in EFs, nodes or connections are deleted. The vulnerability of a network to damage is then assessed by comparing its efficiency after the lesioning to its intact behavior. In an anatomically informed computational model, in line with the DCS view, deletion of nodes did not impair EFs. 


\section{A New Era for Executive Functions}

Despite the appeal of the DCS view as an elegant theoretical solution for the aforementioned problems with centralized EF frameworks, and despite indirect evidence for the viability of DCS as a putative alternative, direct empirical studies of the neurophysiological mechanisms of a DCS governing EFs are scarce. This may be attributed in part to limited awareness of this framework in the field and in part to inherent difficulties in translating DCS concepts, such as the existence of organizing rules, to testable hypotheses. A key challenge is to derive descriptive measures that could provide testable hypothesis of the organizational rules that drive the network towards emergence of EFs. However, one promising approach to investigate EFs from a DCS perspective is the application of methods from network science, primarily graph theory, to neuroimaging data. A DCS conceptualizes executive functioning as an eminent property of multiple interacting elements of the brain. This is analogous to the network science framework, which aims to summarize, via a family of derived metrics, the organizing principles of a set of connected nodes. Hence, the graph theory framework is naturally aligned with a distributed perspective on EFs, with derived metrics potentially capturing the organizing principles or local rules that guide the behavior of the system. The task then becomes to model the brain as a network in which brain areas or constellations act as nodes (i.e. agents) and functional communication among these nodes are represented as edges, and, critically, to relate the static and dynamic features of the resulting network to EFs. In contrast to putting emphasis on the identification of specialized subsystems of EFs that are assumed to exert control over basic processes, a network analytical approach puts focus on how these brain regions or sub-systems (i.e. network nodes) communicate (i.e. are connected) with each other.

From a network perspective, this means that functionally different EFs such as inhibition, and cognitive flexibility can recruit a set of similar or overlapping brain structures (i.e. PFC, ACC, and other regions), but the connectivity patterns within these networks between perceptual input and motor output are functionally different. The connectivity patterns between the involved agents can, in principle, either result in refraining from an action (i.e. inhibition) or shifting to another action (i.e. cognitive flexibility). In other words, we hypothesize that the emergence of a given EF may lie in a given network state, as determined by organizing rules. 
A

$$
\text { Large scale }
$$

communication

(Average path length)

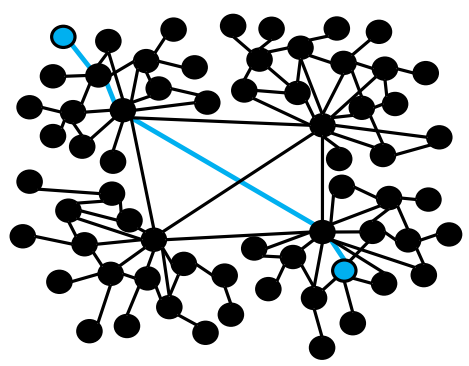

D

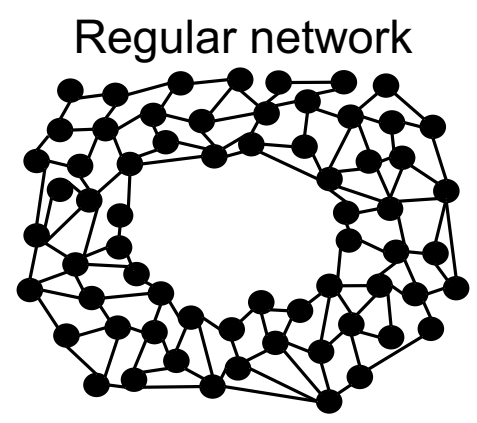

Efficiency: low

Local clustering: high Average path length: high
B

Local clustering (Clustering coefificient)

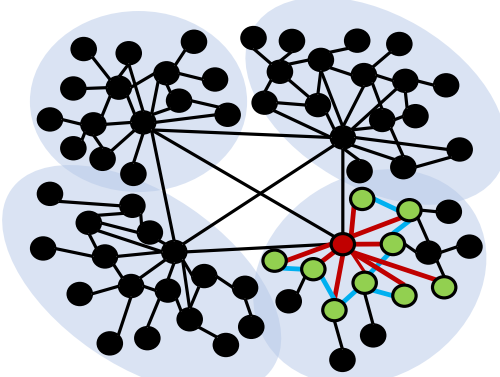

E
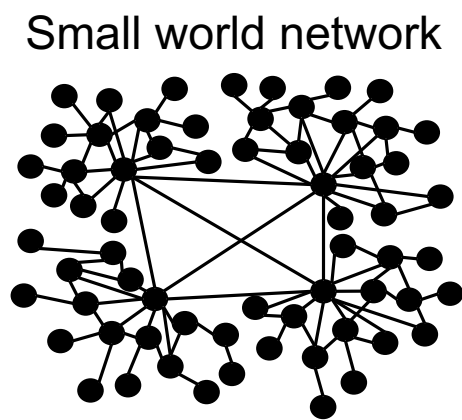

Efficiency: high

Local clustering: high

Average path length: low
C

Hubs of highly connected nodes

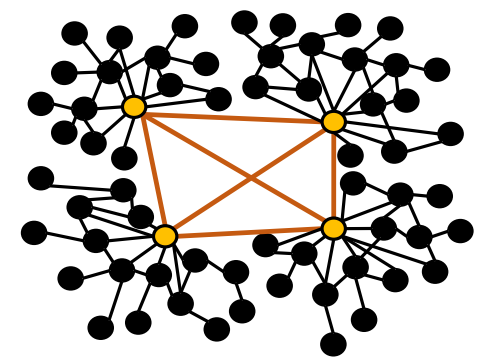

F

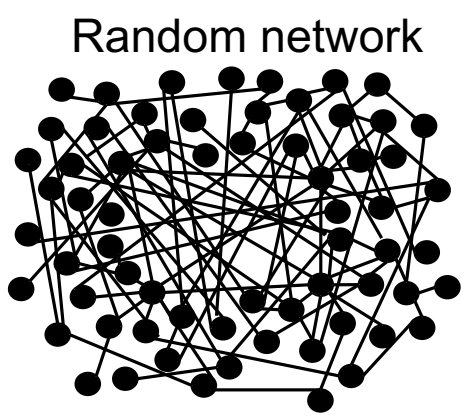

Efficiency: low

Local clustering low Average path length: low

Figure 2. Graph theoretical characteristics of distributed control networks in the brain. Functional integration capacity in neural networks can be quantified by the average path length (A), which describes the average number of edges (marked in blue) to connect each node to any other node in the network (marked in blue). Functional separation capacity in neural networks can be quantified by the clustering coefficient (B), which describes the interconnectivity of neighboring nodes. The clustering coefficient is exemplified in (B), where connections for one node (marked in red) to its immediate neighbors (marked in green) are colored in red and the connections between these neighbors are marked in blue. All real networks lie on a spectrum between very regularly and very randomly connected. Regular networks (D) exhibit a high clustering coefficient and a high average path length. Random networks (F) show a low clustering coefficient and a low average path length. According to Watts and Strogatz ${ }^{205}$, a network shows small-world network properties (E) when it demonstrates a high clustering coefficient as in regular networks and a low average path length as in very random networks. In case a network shows small-world properties, highly connected nodes form hubs that integrate high local connections with long range connections (C).

One important characteristic of networks, and putative organizing rule, is the structural and functional efficiency of its architecture. From an evolutionary perspective, brain networks have been evolved to maximize the diversity of possible functional circuits (i.e. enabling functionally different EFs), while minimizing the diversity of structural circuits (i.e. enabling efficient assembly and encoding) ${ }^{202}$. Thus, the brain seeks towards minimal complexity of neural architecture with maximal richness of function. This may explain why putative brain correlates of specific EFs (i.e. PFC and ACC) have also been associated with other functionally different $\mathrm{EFs}^{39,40,49,50,53,54,66,68,70,72-76,88-93}$. From a graph theoretical 
perspective, the efficiency of large-scale communication between different brain areas is defined by the number of connections necessary to connect all neurons, a measure that is referred to as average synaptic path length (Figure 2A). Additionally, metabolic efficiency in brain networks is realized by predominantly local connectivity ${ }^{34-138} 203$ or local clustering (Figure 2B), which avoids metabolically expensive long-range axonal connections ${ }^{204}$. However, a purely local clustering in the network would dramatically increase the average number of connections necessary to connect all neurons (i.e. the average path length). Thus, two network organization principles-local clustering and average path length -compete with each other to providing network communication that is metabolically efficient (i.e. high local clustering), but also enables effective large-scale communication (i.e. low average path length). Networks, which enable efficient large-scale traffic while maintaining mainly local connectivity are called 'small-world' networks ${ }^{205}$ (Figure 2E). Despite considerable heterogeneity in the methodological approaches, there is an encouraging degree of convergence between small-world properties on the structural ${ }^{202,206-210}$ as well as on the functional brain network level ${ }^{183-185,211-213,213,214}$. Thus, the small-world properties of neural networks may be a key organizing principle that provides a functional link between brain structure and $\mathrm{EFs}^{215}$. Namely, we suggest that EFs are the result of the brain's need to be structurally and functionally efficient. Recent findings highlight that the efficiency of how brain networks communicate (i.e. the small-world network characteristic) is strongly related to the demand on EFs. Differences in small-world properties have been shown for different demands on $\mathrm{EFs}^{216-218}$. Higher demand on inhibition ${ }^{219}$, cognitive flexibility and WM capacity, for instance, have been found to lead to decreased small-world properties in the involved functional networks ${ }^{218}$, suggesting that EFs emerge when the brain is tipped away from a small-world state. This would predict that training EF capacities should lead to more efficient (i.e. more 'small-worldish') functional network communication. Consistent with this prediction, training in EFs such as WM capacity has shown to improve the efficiency of functional networks in the brain ${ }^{220}$.

Of particular interest, small-world architecture results in a system that is neither exclusively top-down in information flow, or exclusively distributed. The system necessitates the existence of so-called hub nodes, that are highly connected and serve a key role in facilitating the balance between high-local clustering and low average path length ${ }^{151}$ (Figure 2C). However, such hub-nodes, due to their highconnectivity, can, in a way, exert control over less-strongly connected nodes along the action cascade and thus may play a key role in driving the functional connectivity state of the system and the emergence of behavior (Figure 2C). Supporting this notion, in a simulated model, deletion of wellconnected nodes produced disruptions of functional connectivity ${ }^{198,221,222}$ consistent with reported effects of focal human brain lesions on $\mathrm{EFs}^{223,224}$. These facts have also led to the proposal that there exists a connective core network, that is a metabolically costly yet highly integratory collective of brain regions, whose anatomical wiring and topological position within the brain network supports brainwide communicability and which exerts control over brain dynamics and cortical states ${ }^{150-152,225-227}$. Here we additionally suggest that such a network drives brain states in observance of or striding towards a small-world state, with no single region acting as a core executive or solely responsible solely for the maintenance of behavioral goals (e.g., PFC). It is sensible to suppose that regions previously associated with EFs (i.e. PFC, ACC, basal ganglia) contribute to this network, though the identity of this network, or networks, remains an open question. A large scale meta-analysis of $\mathrm{fMRI}$ literature has shown that a superordinate fronto-cingulo-parietal network underlies a range of different EFs such as inhibition, flexibility, and working memory ${ }^{228}$, consistent with expectation, but also integrating the inherent biases in published literature. In contrast, in an original research study, Dosenbach et al. (2007), used network analyses on a set of 39 predefined regions of interests (ROIs) to reveal two separate networks of ROls that both showed small-world characteristics: A frontoparietal network that included dIPFC that was more strongly associated with control adaptation and another network including the ACC, that was more strongly associated with WM processes ${ }^{229}$. These data raise the open question of whether there exists a single superordinate EF network, whether multiple networks exist for different EFs or whether, as we suggest here, EFs are purely emergent phenomena with no underlying network, that arise when the brain responds to disturbances of its small-world state. 


\section{Conclusion}

Despite the experimental and theoretical progress in research on EFs, efforts to identify circumscribed brain areas associated with executive functioning have not satisfactorily described the neurophysiological mechanisms that drive EFs. Increasing evidence from neuroimaging suggests instead that the dynamics of a superordinate, spatially distributed brain network, or networks, underlie EFs. A paradigm change from a centralized view, in which single areas exert top down control over the more basic processes they regulate, towards a distributed control system view, in which each brain network agent controls and is being controlled, provide a new perspective on mechanisms of how EFs emerge, with small-world organization a putative driving principle. Emerging studies that leverage network science, and emphasize emergent network dynamics, pave the wave to a new era of EF research.

\section{Acknowledgements}

This work was supported by a grant from the German Research Foundation (DFG) awarded to Sebastian Markett (MA-6792/3-1) and National Institute of Mental Health awarded to Agatha Lenartowicz (R01MH116268).

\section{References}

1. Diamond, A. Executive Functions. Annual Review of Psychology 64, 135-168 (2013).

2. Powell, K. B. \& Voeller, K. K. S. Prefrontal Executive Function Syndromes in Children. Journal of Child Neurology 19, 785-797 (2004).

3. Duncan, J. \& Owen, A. M. Common regions of the human frontal lobe recruited by diverse cognitive demands. Trends in Neurosciences 23, 475-483 (2000).

4. Jurado, M. B. \& Rosselli, M. The Elusive Nature of Executive Functions: A Review of our Current Understanding. Neuropsychology Review 17, 213-233 (2007).

5. Norman, D. A. \& Shallice, T. Attention to action. in Consciousness and self-regulation 1-18 (Springer, 1986).

6. Rabbitt, P. Introduction: Methodologies and models in the study of executive function. Methodology of frontal and executive function 1-38 (1997).

7. $\quad$ Burgess, P. W., Veitch, E., de Lacy Costello, A. \& Shallice, T. The cognitive and neuroanatomical correlates of multitasking. Neuropsychologia 38, 848-863 (2000).

8. Luria, A. R., Karpov, B. A. \& Yarbuss, A. L. Disturbances of active visual perception with lesions of the frontal lobes. Cortex 2, 202-212 (1966).

9. Miyake, A. et al. The unity and diversity of executive functions and their contributions to complex "frontal lobe" tasks: A latent variable analysis. Cognitive psychology 41, 49-100 (2000).

10. Miller, G. A. The magical number seven, plus or minus two: Some limits on our capacity for processing information. Psychological review 63, 81 (1956).

11. Smith, E. E. \& Jonides, J. Storage and executive processes in the frontal lobes. Science 283, 1657-1661 (1999).

12. Baddeley, A. D. \& Hitch, G. Working memory. in Psychology of learning and motivation vol. 8 47-89 (Elsevier, 1974).

13. Cowan, N. An embedded-processes model of working memory. Models of working memory: Mechanisms of active maintenance and executive control 20, 506 (1999).

14. Baddeley, A., Logie, R., Bressi, S., Sala, S. D. \& Spinnler, H. Dementia and working memory. The Quarterly Journal of Experimental Psychology Section A 38, 603-618 (1986). 
15. Jacques, S. \& Zelazo, P. D. Language and the development of cognitive flexibility: implications for theory of mind. in Why Language Matters for Theory of Mind, Apr, 2002, University of Toronto, Toronto, ON, Canada; This chapter originated from the aforementioned conference. (Oxford University Press, 2005).

16. Dajani, D. R. \& Uddin, L. Q. Demystifying cognitive flexibility: implications for clinical and developmental neuroscience. Trends in neurosciences 38, 571-578 (2015).

17. Fischer, R. \& Plessow, F. Efficient multitasking: parallel versus serial processing of multiple tasks. Front Psychol 6, (2015).

18. Wickens, C. D. Processing resources and attention. Multiple-task performance 1991, 3-34 (1991).

19. Kahneman, D. Attention and effort. vol. 1063 (Citeseer, 1973).

20. Broadbent, D. E. Perception and communication. (Elsevier, 2013).

21. Treisman, A. M. \& Gelade, G. A feature-integration theory of attention. Cognitive psychology 12, 97-136 (1980).

22. Deutsch, J. A. \& Deutsch, D. Attention: Some theoretical considerations. Psychological review 70, 80 (1963).

23. Shiffrin, R. M. \& Schneider, W. Controlled and automatic human information processing: II. Perceptual learning, automatic attending and a general theory. Psychological review 84, 127 (1977).

24. Schneider, W. \& Shiffrin, R. M. Controlled and automatic human information processing: I. Detection, search, and attention. Psychological review 84, 1 (1977).

25. Monsell, S. \& Driver, J. Banishing the control homunculus. Control of cognitive processes: Attention and performance XVIII 3-32 (2000).

26. Monsell, S. \& Driver, J. Banishing the control homunculus. Control of cognitive processes: Attention and performance XVIII 3-32 (2000).

27. Logie, R. H. Retiring the central executive. The Quarterly Journal of Experimental Psychology 69, 2093-2109 (2016).

28. Miller, E. K. \& Cohen, J. D. An integrative theory of prefrontal cortex function. Annual Review of Neuroscience 24, 167-202 (2001).

29. Desimone, R. \& Duncan, J. Neural mechanisms of selective visual attention. Annual review of neuroscience 18, 193-222 (1995).

30. Alvarez, J. A. \& Emory, E. Executive Function and the Frontal Lobes: A Meta-Analytic Review. Neuropsychol Rev 16, 17-42 (2006).

31. Newman, S. D., Twieg, D. B. \& Carpenter, P. A. Baseline conditions and subtractive logic in neuroimaging. Human Brain Mapping 14, 228-235 (2001).

32. Friston, K. J. et al. The trouble with cognitive subtraction. Neuroimage 4, 97-104 (1996).

33. Kanwisher, N., McDermott, J. \& Chun, M. M. The fusiform face area: a module in human extrastriate cortex specialized for face perception. Journal of neuroscience 17, 4302-4311 (1997).

34. Houk, J. C. \& Wise, S. P. Distributed modular architectures linking basal ganglia, cerebellum, and cerebral cortex: their role in planning and controlling action. Cerebral cortex 5, 95-110 (1995).

35. Minsky, M. Society of mind. (Simon and Schuster, 1988). 
36. Fodor, J. A. The modularity of mind. (MIT press, 1983).

37. Baddeley, A. Working memory: theories, models, and controversies. Annual review of psychology 63, 1-29 (2012).

38. Botvinick, M. M., Braver, T. S., Barch, D. M., Carter, C. S. \& Cohen, J. D. Conflict monitoring and cognitive control. Psychological review 108, 624 (2001).

39. Botvinick, M. M., Cohen, J. D. \& Carter, C. S. Conflict monitoring and anterior cingulate cortex: an update. Trends in Cognitive Sciences 8, 539-546 (2004).

40. Braver, T. S. The variable nature of cognitive control: A dual-mechanisms framework. Trends Cogn Sci 16, 106-113 (2012).

41. Engle, R. W. \& Kane, M. J. Executive attention, working memory capacity, and a two-factor theory of cognitive control. Psychology of learning and motivation 44, 145-199 (2003).

42. Friston, K. J. Functional and effective connectivity in neuroimaging: a synthesis. Human brain mapping 2, 56-78 (1994).

43. Goldman-Rakic, P. S., Cools, A. R. \& Srivastava, K. The Prefrontal Landscape: Implications of Functional Architecture for Understanding Human Mentation and the Central Executive [and Discussion]. Philosophical Transactions: Biological Sciences 351, 1445-1453 (1996).

44. Goschke, T. \& Bolte, A. Emotional modulation of control dilemmas: The role of positive affect, reward, and dopamine in cognitive stability and flexibility. Neuropsychologia 62, 403-423 (2014).

45. Hommel, B. \& Wiers, R. W. Towards a Unitary Approach to Human Action Control. Trends in Cognitive Sciences 21, 940-949 (2017).

46. Koechlin, E. \& Summerfield, C. An information theoretical approach to prefrontal executive function. Trends in Cognitive Sciences 11, 229-235 (2007).

47. Monsell, S. \& Driver, J. Control of cognitive processes: Attention and performance XVIII. vol. 18 (MIT Press, 2000).

48. Pezzulo, G., Rigoli, F. \& Friston, K. J. Hierarchical Active Inference: A Theory of Motivated Control. Trends in Cognitive Sciences 22, 294-306 (2018).

49. Shenhav, A., Botvinick, M. M. \& Cohen, J. D. The expected value of control: An integrative theory of anterior cingulate cortex function. Neuron 79, 217-240 (2013).

50. Shenhav, A., Cohen, J. D. \& Botvinick, M. M. Dorsal anterior cingulate cortex and the value of control. Nature Neuroscience 19, 1286 (2016).

51. Hazy, T. E., Frank, M. J. \& O'Reilly, R. C. Banishing the homunculus: Making working memory work. Neuroscience 139, 105-118 (2006).

52. Hazy, T. E., Frank, M. J. \& O'Reilly, R. C. Towards an executive without a homunculus: computational models of the prefrontal cortex/basal ganglia system. Philosophical Transactions of the Royal Society B: Biological Sciences (2007).

53. Hare, T. A., Camerer, C. F. \& Rangel, A. Self-Control in Decision-Making Involves Modulation of the vmPFC Valuation System. 324, 4 (2009).

54. Steinbeis, N., Haushofer, J., Fehr, E. \& Singer, T. Development of Behavioral Control and Associated vmPFC-DLPFC Connectivity Explains Children's Increased Resistance to Temptation in Intertemporal Choice. Cerebral Cortex 26, 32-42 (2016). 
55. Sokol-Hessner, P., Hutcherson, C., Hare, T. \& Rangel, A. Decision value computation in DLPFC and VMPFC adjusts to the available decision time: Value computation adjusts to decision time. European Journal of Neuroscience 35, 1065-1074 (2012).

56. Kahnt, T., Heinzle, J., Park, S. Q. \& Haynes, J.-D. Decoding different roles for vmPFC and dIPFC in multi-attribute decision making. NeuroImage 56, 709-715 (2011).

57. Hare, T. A., Hakimi, S. \& Rangel, A. Activity in dlPFC and its effective connectivity to vmPFC are associated with temporal discounting. Frontiers in Neuroscience 8, (2014).

58. Vassena, E., Krebs, R. M., Silvetti, M., Fias, W. \& Verguts, T. Dissociating contributions of ACC and vmPFC in reward prediction, outcome, and choice. Neuropsychologia 59, 112-123 (2014).

59. Lim, S.-L., O’Doherty, J. P. \& Rangel, A. The Decision Value Computations in the vmPFC and Striatum Use a Relative Value Code That is Guided by Visual Attention. Journal of Neuroscience 31, 13214-13223 (2011).

60. Milham, M. P. et al. The relative involvement of anterior cingulate and prefrontal cortex in attentional control depends on nature of conflict. Brain research. Cognitive brain research 12, 467473 (2001).

61. Steinbeis, N., Haushofer, J., Fehr, E. \& Singer, T. Development of Behavioral Control and Associated vmPFC-DLPFC Connectivity Explains Children's Increased Resistance to Temptation in Intertemporal Choice. Cerebral Cortex 26, 32-42 (2016).

62. Aron, A. R., Robbins, T. W. \& Poldrack, R. A. Inhibition and the right inferior frontal cortex. Trends in cognitive sciences 8, 170-177 (2004).

63. Aron, A. R., Robbins, T. W. \& Poldrack, R. A. Inhibition and the right inferior frontal cortex: one decade on. Trends in cognitive sciences 18, 177-185 (2014).

64. Aron, A. R. The neural basis of inhibition in cognitive control. The neuroscientist 13, 214-228 (2007).

65. Hampshire, A., Chamberlain, S. R., Monti, M. M., Duncan, J. \& Owen, A. M. The role of the right inferior frontal gyrus: inhibition and attentional control. Neuroimage 50, 1313-1319 (2010).

66. Ridderinkhof, K. R., Ullsperger, M., Crone, E. A. \& Nieuwenhuis, S. The role of the medial frontal cortex in cognitive control. science 306, 443-447 (2004).

67. Bush, G. et al. Dorsal anterior cingulate cortex: a role in reward-based decision making. Proceedings of the National Academy of Sciences 99, 523-528 (2002).

68. Alexander, W. H. \& Brown, J. W. Medial prefrontal cortex as an action-outcome predictor. Nature Neuroscience 14, 1338-1344 (2011).

69. Egner, T. \& Hirsch, J. Cognitive control mechanisms resolve conflict through cortical amplification of task-relevant information. Nature Neuroscience 8, 1784-1790 (2005).

70. Carter, C. S. \& Veen, V. van. Anterior cingulate cortex and conflict detection: An update of theory and data. Cognitive, Affective, \& Behavioral Neuroscience 7, 367-379 (2007).

71. van Veen, V., Cohen, J. D., Botvinick, M. M., Stenger, V. A. \& Carter, C. S. Anterior Cingulate Cortex, Conflict Monitoring, and Levels of Processing. NeuroImage 14, 1302-1308 (2001).

72. Kerns, J. G. Anterior Cingulate Conflict Monitoring and Adjustments in Control. Science 303, 1023-1026 (2004).

73. MacDonald, A. W., Cohen, J. D., Stenger, V. A. \& Carter, C. S. Dissociating the role of the 
dorsolateral prefrontal and anterior cingulate cortex in cognitive control. Science 288, 1835-1838 (2000).

74. Weissman, D. H. Dorsal Anterior Cingulate Cortex Resolves Conflict from Distracting Stimuli by Boosting Attention toward Relevant Events. Cerebral Cortex 15, 229-237 (2004).

75. Weissman, D. H., Giesbrecht, B., Song, A. W., Mangun, G. R. \& Woldorff, M. G. Conflict monitoring in the human anterior cingulate cortex during selective attention to global and local object features. NeuroImage 19, 1361-1368 (2003).

76. Kondo, H., Osaka, N. \& Osaka, M. Cooperation of the anterior cingulate cortex and dorsolateral prefrontal cortex for attention shifting. NeuroImage 23, 670-679 (2004).

77. Bush, G. et al. Anterior cingulate cortex dysfunction in attention-deficit/hyperactivity disorder revealed by fMRI and the counting stroop. Biological Psychiatry 45, 1542-1552 (1999).

78. Stocco, A., Lebiere, C. \& Anderson, J. R. Conditional routing of information to the cortex: A model of the basal ganglia's role in cognitive coordination. Psychological Review 117, 541-574 (2010).

79. Chakravarthy, V. S., Joseph, D. \& Bapi, R. S. What do the basal ganglia do? A modeling perspective. Biol Cybern 103, 237-253 (2010).

80. Cameron, I. G. M., Watanabe, M., Pari, G. \& Munoz, D. P. Executive impairment in Parkinson's disease: Response automaticity and task switching. Neuropsychologia 48, 1948-1957 (2010).

81. Redgrave, P., Prescott, T. J. \& Gurney, K. The basal ganglia: a vertebrate solution to the selection problem? Neuroscience 89, 1009-1023 (1999).

82. Pauli, W. M., O'Reilly, R. C., Yarkoni, T. \& Wager, T. D. Regional specialization within the human striatum for diverse psychological functions. PNAS 113, 1907-1912 (2016).

83. Aron, A. R. et al. Task-Set Switching Deficits in Early-Stage Huntington's Disease: Implications for Basal Ganglia Function. 15, 17.

84. Poldrack, R. A. Can cognitive processes be inferred from neuroimaging data? Trends in cognitive sciences 10, 59-63 (2006).

85. Poldrack, R. A. \& Wagner, A. D. What can neuroimaging tell us about the mind? Insights from prefrontal cortex. Current Directions in Psychological Science 13, 177-181 (2004).

86. Eisenreich, B. R., Akaishi, R. \& Hayden, B. Y. Control without Controllers: Toward a Distributed Neuroscience of Executive Control. Journal of Cognitive Neuroscience 29, 1684-1698 (2017).

87. Botvinick, M. M. \& Cohen, J. D. The computational and neural basis of cognitive control: charted territory and new frontiers. Cognitive science 38, 1249-1285 (2014).

88. van Veen, V., Cohen, J. D., Botvinick, M. M., Stenger, V. A. \& Carter, C. S. Anterior Cingulate Cortex, Conflict Monitoring, and Levels of Processing. NeuroImage 14, 1302-1308 (2001).

89. Kahnt, T., Heinzle, J., Park, S. Q. \& Haynes, J.-D. Decoding different roles for vmPFC and dlPFC in multi-attribute decision making. NeuroImage 56, 709-715 (2011).

90. Vassena, E., Krebs, R. M., Silvetti, M., Fias, W. \& Verguts, T. Dissociating contributions of ACC and vmPFC in reward prediction, outcome, and choice. Neuropsychologia 59, 112-123 (2014).

91. Milham, M. P. et al. The relative involvement of anterior cingulate and prefrontal cortex in 
attentional control depends on nature of conflict. Brain research. Cognitive brain research 12, 467473 (2001).

92. Bush, G. et al. Anterior cingulate cortex dysfunction in attention-deficit/hyperactivity disorder revealed by fMRI and the counting stroop. Biological Psychiatry 45, 1542-1552 (1999).

93. Lim, S.-L., O'Doherty, J. P. \& Rangel, A. The Decision Value Computations in the vmPFC and Striatum Use a Relative Value Code That is Guided by Visual Attention. Journal of Neuroscience 31, 13214-13223 (2011).

94. Park, H.-J. \& Friston, K. Structural and Functional Brain Networks: From Connections to Cognition. Science 342, 1238411 (2013).

95. Niendam, T. A. et al. Meta-analytic evidence for a superordinate cognitive control network subserving diverse executive functions. Cognitive, Affective, \& Behavioral Neuroscience 12, 241-268 (2012).

96. D'Esposito, M. \& Postle, B. R. The Cognitive Neuroscience of Working Memory. Annu. Rev. Psychol. 66, 115-142 (2015).

97. Banich, M. T. et al. fMRI studies of Stroop tasks reveal unique roles of anterior and posterior brain systems in attentional selection. Journal of cognitive neuroscience 12, 988-1000 (2000).

98. Nicolle, M. M. \& Baxter, M. G. Glutamate receptor binding in the frontal cortex and dorsal striatum of aged rats with impaired attentional set-shifting. European Journal of Neuroscience 18, 3335-3342 (2003).

99. Schroll, H. \& Hamker, F. H. Computational models of basal-ganglia pathway functions: focus on functional neuroanatomy. Front. Syst. Neurosci. 7, (2013).

100. Chang, C., Crottaz-Herbette, S. \& Menon, V. Temporal dynamics of basal ganglia response and connectivity during verbal working memory. Neuroimage 34, 1253-1269 (2007).

101. Voytek, B. \& Knight, R. T. Prefrontal cortex and basal ganglia contributions to visual working memory. Proceedings of the National Academy of Sciences 107, 18167-18172 (2010).

102. McNab, F. \& Klingberg, T. Prefrontal cortex and basal ganglia control access to working memory. Nature neuroscience 11, nn2024 (2007).

103. Frank, M. J., Loughry, B. \& O’Reilly, R. C. Interactions between frontal cortex and basal ganglia in working memory: A computational model. Cognitive, Affective, \& Behavioral Neuroscience 1, 137-160 (2001).

104. Baier, B. et al. Keeping Memory Clear and Stable--The Contribution of Human Basal Ganglia and Prefrontal Cortex to Working Memory. Journal of Neuroscience 30, 9788-9792 (2010).

105. Schroll, H., Vitay, J. \& Hamker, F. H. Working memory and response selection: a computational account of interactions among cortico-basalganglio-thalamic loops. Neural Networks 26, 59-74 (2012).

106. Ashby, F. G., Ell, S. W., Valentin, V. V. \& Casale, M. B. FROST: A distributed neurocomputational model of working memory maintenance. Journal of cognitive neuroscience 17, 1728-1743 (2005).

107. Assaf, M. et al. Abnormal functional connectivity of default mode sub-networks in autism spectrum disorder patients. Neuroimage 53, 247-256 (2010).

108. Baker, J. T. et al. Disruption of Cortical Association Networks in Schizophrenia and Psychotic Bipolar Disorder. JAMA Psychiatry 71, 109 (2014). 
109. Bassett, D. S., Nelson, B. G., Mueller, B. A., Camchong, J. \& Lim, K. O. Altered Resting State Complexity in Schizophrenia. Neuroimage 59, 2196-2207 (2012).

110. Cerliani, L. et al. Increased Functional Connectivity Between Subcortical and Cortical Resting-State Networks in Autism Spectrum Disorder. JAMA Psychiatry 72, 767 (2015).

111. Chai, X. J. et al. Abnormal Medial Prefrontal Cortex Resting-State Connectivity in Bipolar Disorder and Schizophrenia. Neuropsychopharmacology 36, 2009-2017 (2011).

112. Cherkassky, V. L., Kana, R. K., Keller, T. A. \& Just, M. A. Functional connectivity in a baseline resting-state network in autism: NeuroReport 17, 1687-1690 (2006).

113. dos Santos Siqueira, A., Biazoli Junior, C. E., Comfort, W. E., Rohde, L. A. \& Sato, J. R. Abnormal Functional Resting-State Networks in ADHD: Graph Theory and Pattern Recognition Analysis of fMRI Data. BioMed Research International 2014, 1-10 (2014).

114. Du, Y. et al. Interaction among subsystems within default mode network diminished in schizophrenia patients: a dynamic connectivity approach. Schizophr Res 170, 55-65 (2016).

115. Fan, J. et al. Functional deficits of the attentional networks in autism. Brain and Behavior 2, $647-660$ (2012).

116. Fassbender, C. et al. A lack of default network suppression is linked to increased distractibility in ADHD. Brain Res 1273, 114-128 (2009).

117. Franzen, J. D. et al. Atypical coupling between posterior regions of the default mode network in attention-deficit/hyperactivity disorder: a pharmaco-magnetoencephalography study. $J$ Psychiatry Neurosci 38, 333-340 (2013).

118. Garrity, A. G. et al. Aberrant "Default Mode" Functional Connectivity in Schizophrenia. Am J Psychiatry 8 (2007).

119. Hull, J. V. et al. Resting-State Functional Connectivity in Autism Spectrum Disorders: A Review. Frontiers in Psychiatry 7, 205 (2017).

120. Itahashi, T. et al. Altered Network Topologies and Hub Organization in Adults with Autism: A Resting-State fMRI Study. PLoS ONE 9, e94115 (2014).

121. Jafri, M. J., Pearlson, G. D., Stevens, M. \& Calhoun, V. D. A Method for Functional Network Connectivity Among Spatially Independent Resting-State Components in Schizophrenia. Neuroimage 39, 1666-1681 (2008).

122. Jang, J. H. et al. Reduced prefrontal functional connectivity in the default mode network is related to greater psychopathology in subjects with high genetic loading for schizophrenia.

Schizophrenia Research 127, 58-65 (2011).

123. Liddle, E. B. et al. Task-related default mode network modulation and inhibitory control in ADHD: effects of motivation and methylphenidate. Journal of Child Psychology and Psychiatry 52, 761-771 (2011).

124. Lin, H.-Y., Tseng, W. Y. I., Lai, M.-C., Matsuo, K. \& Gau, S. S.-F. Altered resting-state frontoparietal control network in children with attention-deficit/hyperactivity disorder. Journal of the International Neuropsychological Society: JINS 21, 271-284 (2015).

125. Manoliu, A. et al. Aberrant Dependence of Default Mode/Central Executive Network Interactions on Anterior Insular Salience Network Activity in Schizophrenia. Schizophrenia Bulletin 40, 428-437 (2014).

126. Meda, S. A. et al. Multivariate analysis reveals genetic associations of the resting default 
mode network in psychotic bipolar disorder and schizophrenia. Proceedings of the National Academy of Sciences 111, E2066-E2075 (2014).

127. Murias, M., Webb, S. J., Greenson, J. \& Dawson, G. Resting state cortical connectivity reflected in EEG coherence in individuals with autism. Biol Psychiatry 62, 270-273 (2007).

128. Öngür, D. et al. Default Mode Network Abnormalities in Bipolar Disorder and Schizophrenia. Psychiatry Res 183, 59-68 (2010).

129. Orliac, F. et al. Links among resting-state default-mode network, salience network, and symptomatology in schizophrenia. Schizophrenia Research 148, 74-80 (2013).

130. Paakki, J.-J. et al. Alterations in regional homogeneity of resting-state brain activity in autism spectrum disorders. Brain Research 1321, 169-179 (2010).

131. Pomarol-Clotet, E. et al. Failure to deactivate in the prefrontal cortex in schizophrenia: dysfunction of the default mode network? Psychological Medicine 38, 1185-1193 (2008).

132. Roiser, J. P. et al. Dysconnectivity in the Frontoparietal Attention Network in Schizophrenia. Frontiers in Psychiatry 4, (2013).

133. Rotarska-Jagiela, A. et al. Resting-state functional network correlates of psychotic symptoms in schizophrenia. Schizophrenia Research 117, 21-30 (2010).

134. Salgado-Pineda, P. et al. Correlated structural and functional brain abnormalities in the default mode network in schizophrenia patients. Schizophrenia Research 125, 101-109 (2011).

135. Sripada, C. et al. Disrupted network architecture of the resting brain in attentiondeficit/hyperactivity disorder: Disrupted Network Architecture in ADHD. Human Brain Mapping 35, 4693-4705 (2014).

136. Sun, Y., Lim, J., Kwok, K. \& Bezerianos, A. Functional cortical connectivity analysis of mental fatigue unmasks hemispheric asymmetry and changes in small-world networks. Brain and Cognition 85, 220-230 (2014).

137. Swanson, N. et al. Lateral differences in the default mode network in healthy controls and patients with schizophrenia. Human Brain Mapping 32, 654-664 (2011).

138. Tian, L. et al. Enhanced resting-state brain activities in ADHD patients: A fMRI study. Brain and Development 30, 342-348 (2008).

139. Tu, P.-C., Lee, Y.-C., Chen, Y.-S., Li, C.-T. \& Su, T.-P. Schizophrenia and the brain's control network: Aberrant within- and between-network connectivity of the frontoparietal network in schizophrenia. Schizophrenia Research 147, 339-347 (2013).

140. Uddin, L. Q. et al. Network homogeneity reveals decreased integrity of default-mode network in ADHD. Journal of Neuroscience Methods 169, 249-254 (2008).

141. van Buuren, M., Vink, M. \& Kahn, R. S. Default-mode network dysfunction and selfreferential processing in healthy siblings of schizophrenia patients. Schizophrenia Research 142, $237-$ 243 (2012).

142. Wang, K. et al. Selective impairment of attentional networks of orienting and executive control in schizophrenia. Schizophrenia Research 78, 235-241 (2005).

143. Weng, S.-J. et al. Alterations of Resting State Functional Connectivity in the Default Network in Adolescents with Autism Spectrum Disorders. Brain Res 1313, 202 (2010).

144. Whitfield-Gabrieli, S. et al. Hyperactivity and hyperconnectivity of the default network in 
schizophrenia and in first-degree relatives of persons with schizophrenia. Proceedings of the National Academy of Sciences 106, 1279-1284 (2009).

145. Wilson, T. W. et al. Broadband neurophysiological abnormalities in the medial prefrontal region of the default-mode network in adults with ADHD. Human Brain Mapping n/a-n/a (2011) doi:10.1002/hbm.21459.

146. Woodward, N. D., Rogers, B. \& Heckers, S. Functional resting-state networks are differentially affected in schizophrenia. Schizophr Res 130, 86-93 (2011).

147. Barnard, P. J. \& Bowman, H. Rendering information processing models of cognition and affect compu- tationally explicit: distributed executive control and the deployment of attention. 35 .

148. Vandierendonck, A. A working memory system with distributed executive control.

Perspectives on Psychological Science 11, 74-100 (2016).

149. Margulies, D. S. et al. Situating the default-mode network along a principal gradient of macroscale cortical organization. Proceedings of the National Academy of Sciences 113, 12574-12579 (2016).

150. van den Heuvel, M. P., Kahn, R. S., Goñi, J. \& Sporns, O. High-cost, high-capacity backbone for global brain communication. Proceedings of the National Academy of Sciences 109, 11372-11377 (2012).

151. van den Heuvel, M. P. \& Sporns, O. Network hubs in the human brain. Trends in cognitive sciences 17, 683-696 (2013).

152. Collin, G., Sporns, O., Mandl, R. C. \& van den Heuvel, M. P. Structural and functional aspects relating to cost and benefit of rich club organization in the human cerebral cortex. Cerebral cortex $\mathbf{2 4}$, 2258-2267 (2014).

153. James, L. \& Rumelhart, D. E. PARALLEL DISTRIBUTED PROCESSING, EXPLORATIONS IN THE MICROSTRUCTURE OF COGNITION: VOL 2, PSYCHOLOGICAL AND BIOLOGICAL MODELS. (1986).

154. Hopfield, J. J. Neural networks and physical systems with emergent collective computational abilities. Proceedings of the national academy of sciences 79, 2554-2558 (1982).

155. McClelland, J. L. et al. Letting structure emerge: connectionist and dynamical systems approaches to cognition. Trends in cognitive sciences 14, 348-356 (2010).

156. Mitchell, M. Complexity: A guided tour. (Oxford University Press, 2009).

157. Couzin, I. D. Collective cognition in animal groups. Trends in cognitive sciences $\mathbf{1 3}, 36-43$ (2009).

158. Passino, K. M., Seeley, T. D. \& Visscher, P. K. Swarm cognition in honey bees. Behavioral Ecology and Sociobiology 62, 401-414 (2008).

159. Strandburg-Peshkin, A., Farine, D. R., Couzin, I. D. \& Crofoot, M. C. Shared decision-making drives collective movement in wild baboons. Science 348, 1358-1361 (2015).

160. Couzin, I. D. \& Krause, J. Self-organization and collective behavior in vertebrates. Advances in the Study of Behavior 32, 10-1016 (2003).

161. LeCun, Y., Bengio, Y. \& Hinton, G. Deep learning. nature 521, 436-444 (2015).

162. Sleezer, B. J. \& Hayden, B. Y. Differential contributions of ventral and dorsal striatum to early and late phases of cognitive set reconfiguration. Journal of cognitive neuroscience 28, 1849-1864 
(2016).

163. Cisek, P. \& Kalaska, J. F. Neural mechanisms for interacting with a world full of action choices. Annual review of neuroscience 33, 269-298 (2010).

164. Postle, B. R. Working memory as an emergent property of the mind and brain. Neuroscience 139, 23-38 (2006).

165. Awh, E. \& Jonides, J. Overlapping mechanisms of attention and spatial working memory. Trends in cognitive sciences 5, 119-126 (2001).

166. Lee, S.-H. \& Baker, C. I. Multi-Voxel Decoding and the Topography of Maintained Information During Visual Working Memory. Front. Syst. Neurosci. 10, (2016).

167. Buzsáki, G. Rhythms of the brain. (Oxford Univ. Press, 2006).

168. Chance, F. S., Abbott, L. F. \& Reyes, A. D. Gain Modulation from Background Synaptic Input. Neuron 35, 773-782 (2002).

169. Salinas, E. \& Thier, P. Gain modulation: a major computational principle of the central nervous system. Neuron 27, 15-21 (2000).

170. Donner, T. H. \& Nieuwenhuis, S. Brain-wide gain modulation: the rich get richer. Nat Neurosci 16, 989-990 (2013).

171. Saalmann, Y. B. \& Kastner, S. Gain control in the visual thalamus during perception and cognition. Curr Opin Neurobiol 19, 408-414 (2009).

172. Abbott, L. F. Synaptic Depression and Cortical Gain Control. Science 275, 221-224 (1997).

173. Desimone, R. Visual attention mediated by biased competition in extrastriate visual cortex. Philosophical Transactions of the Royal Society of London. Series B: Biological Sciences 353, 12451255 (1998).

174. Sumpter, D. J. The principles of collective animal behaviour. Philosophical transactions of the royal society B: Biological Sciences 361, 5-22 (2006).

175. Achard, S. A Resilient, Low-Frequency, Small-World Human Brain Functional Network with Highly Connected Association Cortical Hubs. Journal of Neuroscience 26, 63-72 (2006).

176. Bassett, D. S. \& Bullmore, E. Small-World Brain Networks. The Neuroscientist 12, 512-523 (2006).

177. Bullmore, E. \& Sporns, O. Complex brain networks: graph theoretical analysis of structural and functional systems. Nature Reviews Neuroscience 10, 186-198 (2009).

178. Ahmadlou, M., Ahmadi, K., Rezazade, M. \& Azad-Marzabadi, E. Global organization of functional brain connectivity in methamphetamine abusers. Clinical Neurophysiology 124, 1122-1131 (2013).

179. Yuan, K. et al. Altered small-world brain functional networks and duration of heroin use in male abstinent heroin-dependent individuals. Neuroscience Letters 477, 37-42 (2010).

180. Wang, Z. et al. A Hyper-connected but Less Efficient Small-world Network in the SubstanceDependent Brain. Drug Alcohol Depend 152, 102-108 (2015).

181. Ahmadlou, M., Adeli, H. \& Adeli, A. Graph theoretical analysis of organization of functional brain networks in ADHD. Clin EEG Neurosci 43, 5-13 (2012).

182. Liu, T., Chen, Y., Lin, P. \& Wang, J. Small-World Brain Functional Networks in Children 
With Attention-Deficit/Hyperactivity Disorder Revealed by EEG Synchrony. Clin EEG Neurosci 46, 183-191 (2015).

183. Wang, L. et al. Altered small-world brain functional networks in children with attentiondeficit/hyperactivity disorder. Human Brain Mapping 30, 638-649 (2009).

184. Xia, S., Foxe, J. J., Sroubek, A. E., Branch, C. \& Li, X. Topological organization of the "small-world" visual attention network in children with attention deficit/hyperactivity disorder (ADHD). Front. Hum. Neurosci. 8, (2014).

185. Liu, Y. et al. Disrupted small-world networks in schizophrenia. Brain 131, 945-961 (2008).

186. Micheloyannis, S. et al. Small-world networks and disturbed functional connectivity in schizophrenia. Schizophrenia Research 87, 60-66 (2006).

187. Rubinov, M. et al. Small-world properties of nonlinear brain activity in schizophrenia. Human Brain Mapping 30, 403-416 (2009).

188. Shim, M., Kim, D.-W., Lee, S.-H. \& Im, C.-H. Disruptions in small-world cortical functional connectivity network during an auditory oddball paradigm task in patients with schizophrenia.

Schizophrenia Research 156, 197-203 (2014).

189. Choi, J. W. et al. Abnormal Sleep Delta Rhythm and Interregional Phase Synchrony in Patients with Restless Legs Syndrome and Their Reversal by Dopamine Agonist Treatment. J Clin Neurol 13, 340 (2017).

190. Barttfeld, P. et al. A big-world network in ASD: dynamical connectivity analysis reflects a deficit in long-range connections and an excess of short-range connections. Neuropsychologia 49, 254-263 (2011).

191. Frantzidis, C. A. et al. Functional disorganization of small-world brain networks in mild Alzheimer's Disease and amnestic Mild Cognitive Impairment: an EEG study using Relative Wavelet Entropy (RWE). Front Aging Neurosci 6, (2014).

192. Stam, C., Jones, B., Nolte, G., Breakspear, M. \& Scheltens, P. Small-World Networks and Functional Connectivity in Alzheimer's Disease. Cerebral Cortex 17, $92-99$ (2006).

193. Vecchio, F. et al. Cortical connectivity and memory performance in cognitive decline: A study via graph theory from EEG data. Neuroscience 316, 143-150 (2016).

194. Wei, L., Li, Y., Yang, X., Xue, Q. \& Wang, Y. Altered characteristic of brain networks in mild cognitive impairment during a selective attention task: An EEG study. International Journal of Psychophysiology 98, 8-16 (2015).

195. Zeng, K. et al. Complex network analysis of resting state EEG in amnestic mild cognitive impairment patients with type 2 diabetes. Front Comput Neurosci 9, 133 (2015).

196. Berman, B. D. et al. Levodopa modulates small-world architecture of functional brain networks in Parkinson's disease: Levodopa Modulates Brain Networks IN PD. Mov Disord. 31, 16761684 (2016).

197. Lebedev, A. V. et al. Large-scale resting state network correlates of cognitive impairment in Parkinson's disease and related dopaminergic deficits. Front. Syst. Neurosci. 8, (2014).

198. Honey, C. J. \& Sporns, O. Dynamical consequences of lesions in cortical networks. Hum. Brain Mapp. 29, 802-809 (2008).

199. Kaiser, M., Martin, R., Andras, P. \& Young, M. P. Simulation of robustness against lesions of cortical networks. European Journal of Neuroscience 25, 3185-3192 (2007). 
200. Ravasz, E. \& Barabási, A.-L. Hierarchical organization in complex networks. Physical Review E 67, 026112 (2003).

201. Sporns, O. Small-world connectivity, motif composition, and complexity of fractal neuronal connections. Biosystems 85, 55-64 (2006).

202. Sporns, O. \& Kötter, R. Motifs in Brain Networks. PLoS Biology 2, e369 (2004).

203. Yoshimura, Y., Dantzker, J. L. M. \& Callaway, E. M. Excitatory cortical neurons form finescale functional networks. Nature 433, 868-873 (2005).

204. Kalisman, N., Silberberg, G. \& Markram, H. The neocortical microcircuit as a tabula rasa. Proceedings of the National Academy of Sciences of the United States of America 102, 880-885 (2005).

205. Watts, D. J. \& Strogatz, S. H. Collective dynamics of 'small-world' networks. Nature 393, 440-442 (1998).

206. Iturria-Medina, Y., Sotero, R. C., Canales-Rodríguez, E. J., Alemán-Gómez, Y. \& MelieGarcía, L. Studying the human brain anatomical network via diffusion-weighted MRI and Graph Theory. Neuroimage 40, 1064-1076 (2008).

207. Iturria-Medina, Y. et al. Characterizing brain anatomical connections using diffusion weighted MRI and graph theory. Neuroimage 36, 645-660 (2007).

208. Hagmann, P. et al. Mapping Human Whole-Brain Structural Networks with Diffusion MRI. PLoS ONE 2, e597 (2007).

209. Chen, Z. J., He, Y., Rosa-Neto, P., Germann, J. \& Evans, A. C. Revealing Modular Architecture of Human Brain Structural Networks by Using Cortical Thickness from MRI. Cerebral Cortex 18, 2374-2381 (2008).

210. He, Y., Chen, Z. J. \& Evans, A. C. Small-World Anatomical Networks in the Human Brain Revealed by Cortical Thickness from MRI. Cerebral Cortex 17, 2407-2419 (2007).

211. Ferrarini, L. et al. Hierarchical functional modularity in the resting-state human brain. Human Brain Mapping 30, 2220-2231 (2009).

212. Meunier, D., Achard, S., Morcom, A. \& Bullmore, E. Age-related changes in modular organization of human brain functional networks. Neuroimage 44, 715-723 (2009).

213. Salvador, R. Neurophysiological Architecture of Functional Magnetic Resonance Images of Human Brain. Cerebral Cortex 15, 1332-1342 (2004).

214. Schwarz, A. J., Gozzi, A. \& Bifone, A. Community structure and modularity in networks of correlated brain activity. Magnetic resonance imaging 26, 914-920 (2008).

215. Beste, C. et al. How minimal variations in neuronal cytoskeletal integrity modulate cognitive control. NeuroImage 185, 129-139 (2019).

216. Zink, N., Stock, A.-K., Colzato, L. \& Beste, C. Evidence for a neural dual-process account for adverse effects of cognitive control. Brain Struct Funct 1-17 (2018) doi:10.1007/s00429-018-1694-1.

217. Beste, C. et al. How minimal variations in neuronal cytoskeletal integrity modulate cognitive control. NeuroImage 185, 129-139 (2019).

218. Wolff, N., Zink, N., Stock, A.-K. \& Beste, C. On the relevance of the alpha frequency oscillation's small-world network architecture for cognitive flexibility. Scientific Reports 7, (2017).

219. Hong, X., Liu, Y., Sun, J. \& Tong, S. Age-Related Differences in the Modulation of Small- 
World Brain Networks during a Go/NoGo Task. Front. Aging Neurosci. 8, (2016).

220. Langer, N., von Bastian, C. C., Wirz, H., Oberauer, K. \& Jäncke, L. The effects of working memory training on functional brain network efficiency. Cortex 49, 2424-2438 (2013).

221. Jirsa, V., Sporns, O., Breakspear, M., Deco, G. \& McIntosh, A. R. Towards the virtual brain: network modeling of the intact and the damaged brain. Archives italiennes de biologie 148, 189-205 (2010).

222. Falcon, M. I. et al. The Virtual Brain: modeling biological correlates of recovery after chronic stroke. Frontiers in neurology 6, 228 (2015).

223. He, B. J., Shulman, G. L., Snyder, A. Z. \& Corbetta, M. The role of impaired neuronal communication in neurological disorders: Current Opinion in Neurology 20, 655-660 (2007).

224. He, B. J. et al. Breakdown of Functional Connectivity in Frontoparietal Networks Underlies Behavioral Deficits in Spatial Neglect. Neuron 53, 905-918 (2007).

225. de Reus, M. A. \& van den Heuvel, M. P. Simulated rich club lesioning in brain networks: a scaffold for communication and integration? Front. Hum. Neurosci. 8, (2014).

226. Senden, M., Reuter, N., van den Heuvel, M. P., Goebel, R. \& Deco, G. Cortical rich club regions can organize state-dependent functional network formation by engaging in oscillatory behavior. NeuroImage 146, 561-574 (2017).

227. Shine, J. M., Aburn, M. J., Breakspear, M. \& Poldrack, R. A. The modulation of neural gain facilitates a transition between functional segregation and integration in the brain. eLife 7, e31130 (2018).

228. Niendam, T. A. et al. Meta-analytic evidence for a superordinate cognitive control network subserving diverse executive functions. Cognitive, Affective, \& Behavioral Neuroscience 12, 241-268 (2012).

229. Dosenbach, N. U. F. et al. Distinct brain networks for adaptive and stable task control in humans. Proceedings of the National Academy of Sciences 104, 11073-11078 (2007).

230. Eisenreich, B. R., Akaishi, R. \& Hayden, B. Y. Control without Controllers: Toward a Distributed Neuroscience of Executive Control. Journal of Cognitive Neuroscience 29, 1684-1698 (2017). 